\title{
How to write a scientific masterpiece
}

\author{
Ushma S. Neill \\ Executive Editor, The Journal of Clinical Investigation
}

\begin{abstract}
I've been asked several times to give talks about various aspects of the scientific publishing enterprise, and sometimes to comment specifically on how to write a manuscript that will have maximal impact. While many in my audiences have felt that my presentations are designed for students and trainees, $I$ hope everyone listens, as sometimes even established scientists are prone to making mistakes. I hope here to outline a few pointers that will help your manuscripts skate through the submission and peer review process. Some points may be elementary, but all bear repeating.
\end{abstract}

\section{Before you start writing}

It goes without saying that you need to be realistic about which journal to send your work to in the first place. Our particular goal at the JCI is to publish basic biological findings and translational studies that have clear biomedical interest and implications for the treatment of human diseases and represent a novel conceptual advance; I realize this is a very broad statement. What I mean is that to present an advance in the understanding of a disease mechanism or pathogenesis usually involves in vivo models or clinical samples. Studies carried out primarily in vitro are unlikely to be competitive at the JCI, something I learned when I submitted my entirely in vitro work to the JCI as a graduate student (I know how rejection feels, too). Take a good look at the hypothesis you've sought to prove, the experiments performed, and their implications before deciding which journal is the best fit for your manuscript. Consider where you find most of the papers that are relevant to your own work - perhaps in those journals your work will gain the most exposure and appreciation.

Another important early consideration has to do with authorship. I encourage you to openly discuss from the start who is to be an author; the Scientist recently had a very interesting open debate about what defines authorship (1). We, however, have established no hard and fast rules about what merits authorship versus acknowledgment or how to determine the order of authors; the JCI will not get involved in authorship disputes, and you need to determine this on your own. We encourage you to keep track of who did what and also to discuss who gets to write the paper, as this person is usually the first author. You should also consider shared first authorship and/or senior authorship if the situation merits this designation, as most journals (including the JCI) will allow this.

Once you start to write, do not forget the importance of a good cover letter; one that is well worded can be a very important weapon in your arsenal. After the title (more on that in a moment), this is your chance to set the stage for what is to come, your opportunity to really sell your findings and their importance. Papers submitted with and without extensive cover letters are read equally thoroughly, but a good cover letter helps the editor understand the implications of the manuscript and appreciate how you, the author, believe it fits into the broader field and makes a significant contribution. Remember that there is an art to writing a good cover letter: it should be a maximum of 4-5 paragraphs in length - any more and we question why you are arguing so vehemently (heading off questions asked by previous referees? hinting at a fundamental flaw?); much less

Citation for this article: J. Clin. Invest. 117:3599-3602 (2007). doi:10.1172/JCI34288. and it is probably not sufficient to communicate your message. What is to be contained in these precious few paragraphs? First, introduce the study and list the authors. The middle paragraphs should be dedicated to explaining the basic premise of your study and why the findings are interesting and novel. In the case of the JCI, you should also note what clinical implication your work may have. You may also use a paragraph in the cover letter to suggest referees and also to explain any exclusions that you want the editors to respect; the JCI will only entertain 2 exclusions - any more will be up to the discretion of the editors (more on this later). End the cover letter by declaring any conflicts of interest that you may have (make sure to look up the specific conflict-of-interest policy for the journal to which you are submitting, as different journals have different rules about what you must declare); indicating that the findings are as yet unpublished (or where, in abstract form, they may have been referred to); and also verifying that the work is not currently under consideration at any other journal. A last point that I cannot emphasize heavily enough: make sure to address your cover letter to the correct journal, and also make sure the date listed on the letter is current. We understand where we fall in the hierarchy of journals, but at least give us the delusion that we were your first choice. I also note here that it is a good idea to change the names of your files and the headers/footers from "ScienceSubmission07" to "JCISubmission07" prior to uploading your files to our database. The realization that a competitor rejected the paper can result in a negative predisposition.

Make sure to format your paper according to the specific journal's instructions. In addition to the cover letter faux pas, a giveaway that a paper was previously submitted elsewhere is when the sections are not in the proper order or the references are not properly formatted (e.g., in alphabetical order as opposed to numbered). Other details, like appropriate italicization of terms (gene names and mRNAs, but not gene products or terms such as in vivo) should be kept in mind.

\section{Writing the paper}

Title. Start with an appropriate title, not one that inflates the relevance of your findings and not one that claims to cure cancer and the common cold. And it need not be long: 15 words should suffice (the limit at the JCI). No jargon should be used in the title, or anywhere else in the text for that matter; keep in mind that you want to attract the most diverse readership to your articles (particularly important at general biomedical journals such as the $J C I$ ), and if people can't understand your title, they will not read your paper. Avoid using excess punctuation: i.e., no colons, excess 
commas (parentheticals), "and terms in quotes." And try to get the species studied into the title; this is sometimes complicated if multiple species are used, but it allows readers to immediately put the work in its appropriate context. As examples, I offer the succinct title from an article published in 2006, "Activated macrophages are essential in a murine model for T cell-mediated chronic psoriasiform skin inflammation" and, as a corollary, a title from 1962, "Pathogenesis of the coagulation defect developing during pathological plasma proteolytic ('fibrinolytic') states: the significance of fibrinogen proteolysis and circulating fibrinogen breakdown products." While I am sure this is informative to some readers, I fell asleep halfway through the second line and have no idea whether this was done in vitro or in vivo or in what species.

Abstract. The abstract is your hook, the most important information readers use when deciding whether to abandon your article or read it more in depth. It pays to make it easy to understand and broadly appealing; informative but not too detailed. What is the formula for such a masterpiece? Here is one that we try to follow as best we can: first, start with a sentence or two that frames the work. Introduce the disease and system you are studying and indicate what was previously unknown - framing why we are here now. Move on to the major finding, then spend a few sentences detailing the steps and mechanisms uncovered. Make sure to indicate the species studied, especially when this changes with different experiments. End the abstract with a sentence or two indicating the implications of the work without inflating the relevance.

In addition, in the abstract, use the present tense to refer to facts that have already been established in the field. Then move on to the past tense to describe the findings from the current study. This way it is easy for readers to parse what was done in the current study and what was previously known. Try to avoid the passive voice if you can. Near the beginning, it is helpful to distinguish between what was already published and what is currently shown by using a phrase akin to, "Here, we showed ..."

Results. As for writing the rest of the paper, my suggestions will largely be a summary of the points perfectly expressed by William Wells in an article from the Journal of Cell Biology on how to write a scientific paper (2). I suggest you begin writing the Results section before other sections, as the experiments described will then dictate what needs to be detailed in Methods, and also what needs to be introduced and thoroughly discussed; not every experiment performed needs to make it into the paper. You can choose to present the experiments either in the chronological order in which you performed them, if you happen to have been that prescient about what the results would have been or, rather, in an order wherein the most important result is presented first, and the supporting and mechanistic data follow. Bear in mind that too much data can be distracting - make use of the supplemental data section should the need arise.

In the Results section, you should describe the data succinctly and take pains to indicate statistical differences; save most interpretation for the Discussion. A certain amount of interpretation is, of course, encouraged when describing the logical progression of experiments: "because A resulted in $\mathrm{B}$, which is in the cascade of C (citation), we decided to see whether A was connected to C; therefore, we subjected D to E."

Discussion. You should start the Discussion section with a summary of the research presented and the implications for broader application. The Discussion should not simply be a recapitulation of the Results, but rather an interpretation of how each of the experiments supported your central hypothesis. This is your chance to add in supporting findings from the literature and to argue why your study is a conceptual advance over those studies. The end of this section should also touch on the areas left to investigate and mysteries that remain. You may choose to add a schematic figure to the Discussion if the signaling cascades studied are particularly complex; however, try not to use lightning bolts and scissors within these schematics.

Introduction. The structure of the Introduction is somewhat different from that of the Discussion, and the purpose is also distinct, but they are similar in that they are the sections where you can argue your hypothesis. Start the Introduction by providing the background for the field - how does it link to your particular signaling cascade/polymorphism/technique of interest and what was heretofore unknown - alongside your hypothesis for how you will fill that gap in knowledge. Make sure to furnish relevant references in the Introduction, as we do not want to find a reference at the end of the Discussion that shows your whole study was simply a recapitulation of another study. Also make sure to add all salient references - we can use PubMed as easily as you can, and nothing will ensure a faster rejection than finding that you have selectively referenced your article. Finish the Introduction with a short paragraph detailing what you are about to show.

Methods. Provide enough detail so that any reader would be able to repeat your experiment based solely on the information provided in the Methods. Treat it like a recipe: give concentrations and amounts used, temperatures and durations of experiments, $n$ values and details of equipment used, and cell/animal sources. You need to include a separate paragraph detailing your statistical methods. You also need to explicitly state which institutional authority granted approval for the animal experiments, as a simple statement that you followed the rules of animal care is not sufficient. If the experiments were done in humans, you need to declare that you had written informed consent. Prior to submission, you must submit original nucleotide or amino acid sequence data to GenBank, the European Molecular Biology Laboratory (EMBL), the DNA Data Bank of Japan (DDBJ), or another appropriate, identified, publicly available database in general use in the field that gives free access to researchers from the date of publication. Accession numbers should appear in the manuscript.

Figures. A reader familiar with the topic should be able to understand your work solely by looking at the figures and legends. Title your figures in the legend as you would a subheading in the text. Also limit the figure legends to 250 words or less. They should include no methodology, but make sure to list $n$ values. Keep highresolution pictures for ultimate reproduction and publication. Keep them well organized as well, so there is no confusion if/when you are questioned about your figures. Try to provide quantification of blots and of histology when possible. When presenting histograms, use black and white bars, then gray/shaded bars, as opposed to colored bars. Keep in mind that approximately $10 \%$ of the male population is red/green colorblind, and try to avoid using red and green bars next to each other. Avoid gratuitous use of color for decorative effect, boxes around graphs and figures, and small type and symbols on large graphs. Type appearing within figures (axis labels, for example) should be of sufficient size and contrast to retain clarity if reduced in size. When using scale bars, please define scales in figure legends, not in the figures themselves. Whenever possible, do not place labels over any part of a color figure. Provide a key to any symbols used. Do not overfill a figure with too many panels. 


\section{The proper way to present a point-by-point response}

Referee Point 1: The authors make the point that A shows B through $\mathrm{C}$ in $\mathrm{D}$ cells, but they do not provide any evidence to show that $B$ works through $\mathrm{C}$ in an in vivo model of $\mathrm{E}$ syndrome or in clinical samples from patients with $\mathrm{E}$ syndrome. Demonstrating $B$ functions through $\mathrm{C}$ in the $\mathrm{F}$ model of $\mathrm{E}$ syndrome would be required at a minimum.

Response: We thank this reviewer for his/her critical and helpful evaluation of our manuscript. In response to the reviewer's critique, our manuscript has undergone a major revision. In Figure 4 we have added new data in the F model of E syndrome that demonstrate that B goes through C. In Figure 5 we investigated B expression in a case series of biopsies from patients with E syndrome to confirm the result in human samples.

\section{Other considerations}

Peer review. You have the option to suggest as well as exclude referees, and most journals welcome suggestions. As the author, you may be equally or better placed than the editors to know who is best qualified to evaluate and recognize the impact of your work. It is appreciated if you provide the contact information for at least five referees. Suggest established investigators with a broad knowledge of the field and those with the technical expertise to evaluate your experimental approach. Also look at the masthead of the journal to which you are submitting your manuscript to see whether there is a board of consulting editors or the like, as these are often the people who are most often employed as consultants. Do not suggest researchers that are in your department, institution, or company - even if they are at another campus. Friends, recent (less than 3 years) coauthors, and collaborators are also not appropriate to suggest. This includes those you acknowledge in the manuscript for providing reagents or critiques.

As for exclusions, we understand that you may have valid reasons for keeping sensitive results out of competitors' hands, and we will respect 2 exclusions as long as you give a short description of why: financial conflict, known bias, etc. If you try to exclude everyone in your field or an entire institution, you will appear paranoid, and we will question whether the work is controversial (which is fine) or totally off kilter.

How do the editors choose referees? A quick spin through PubMed is often helpful, and we look at the authors of the most salient references while keeping in mind the authors' suggestions and exclusions. We use those with technical expertise and broad understanding of the field, those who are efficient, fair-minded, and constructive. We also try to use referees who have either previously served as referees or have been authors for the journal, as they are familiar with our standards. We have also established a conflict-of-interest policy for reviewers and ask that our referees adhere to it (3).

Decisions. Please don't call or write to the editors daily to inquire as to the status of your manuscript; we do not sit on decisions, and you will know as soon as we have properly discussed your article. Remember that very few papers are accepted upon initial submission (I can remember only one in the past seven years), and on average it takes 1-2 revisions before a work is acceptable. If the paper is rejected, don't be discouraged, as even Nobel laureates get rejection letters - Rosalyn Yalow famously showed a rejection letter from the JCI in her acceptance speech for the 1977 Nobel Prize in Physiology or Medicine (4). And please do not take a decision letter personally - sending rejection letters brings no editor any particular joy.

If you choose to submit elsewhere, carefully consider your second choice, and remember to change your cover letter. You should recognize and fix the major flaws before submitting to another journal and make a modest effort to incorporate the referee suggestions, as it is always possible the same referee will see it again. Peer review should help improve the paper.

Revision. Gratifyingly, not all papers are rejected by the JCI. Read your letters carefully to determine whether there is hope and if the editors specifically mentioned anything that would be absolutely required in a revision. If an editor customizes the letter to underscore a particular point, it is meant to assist you by identifying particularly key issues, but at the same time, it is not a free pass to ignore the other referee comments, and you must provide a robust response to those points in addition to the ones the editor outlines. Endeavor to revise and resubmit within three months, but take all the time you need, as a hastily prepared, insufficient revision is likely to be rejected. However, regardless of whether a manuscript is returned to us within two days or three months, the editors will determine whether the findings are still novel: if any manuscripts appear within the literature that compromise the novelty of your work, we are under no obligation to consider the work further.

When revising your manuscript, do not cherry pick which points you wish to respond to and which you don't. You need to address all the referee and editor comments with new experimental data, editorial changes, or a convincing counterargument when appropriate. The referees and editors will notice if you have skipped a comment in your point-by-point response. Also make sure to actually incorporate the data/text into the article and not just discuss it in the point by point; again, we will notice if it is not there.

In the point-by-point letter, always be respectful and polite, even if it hurts. As the adage goes, you will catch a lot more flies with honey than with vinegar. In the first few lines of the letter to be transmitted to the referees, thank him/her for the insightful and helpful comments. Then break down each of their points (in bold, italicized, or colored font) and provide a reply to each point (see The proper way to present a point-by-point response) in plain, unformatted text (or vice versa, with unformatted text for the referee and bold text for your response). This may seem elementary, but we have received any number of permutations: authors provide narrative responses (without detailing which of the referee's points is being addressed) and replies like "1. Change made. 2. Change made" when the referee's points 1 and 2 concerned creation of new animal models. It is worth being explicit about the reasons behind the change and what exactly you did, and where in the manuscript those changes can be found. Also, if multiple referees ask for the same changes, repeat yourself in the response to each referee (unless the response is very long) - it is irritating when an author replies "see change indicated in response to point 1 of referee b." This need not 
be a long letter, but space is not limiting, and this is your chance to impress the referee that you have taken their comments seriously. Do the work for them. You may also choose to highlight or underline within the text the passages that have changed, and while this can be distracting if the changes are substantial, it may also assist the referees and the editors in rapidly identifying the revisions.

If you cannot accommodate the demands of the referee, thank the referee for the suggestion, but offer an explanation as to why the experiments are not possible at this time, or perhaps why they are beyond the scope of the current paper. This argument may not always work, as the editors may repeat their request for a specific experiment, but if an experiment is simply impossible, give a short, convincing explanation.

Appealing the decision. If you receive a rejection letter, put it away for a minimum of 24 hours. Knee-jerk responses are rarely rational or well constructed. Also, if you are making fun of us, take special care to ensure you click "forward" and not "reply" to the decision letter, as no matter how amusing I find these letters, they are not usually meant for our eyes (the most entertaining of these e-mails said I deserved a spanking). Determine whether a rebuttal letter is truly your best option, as you may not submit the work elsewhere while the rebuttal is still being considered. But editors and referees do make mistakes, and we are willing to overrule specific points, especially if there were factual errors in the reviews. While I have no statistics on how many rebuttals are successful, I note that the large majority of decisions are not overturned.

What helps in a rebuttal letter? As in the point-by-point letter, be polite, even if you disagree. Do not guess at the referee's identity, as most of the time you are incorrect, and we will not reveal any information related to who did or did not provide input into the decision; it is helpful, however, to have specific evidence if you feel a referee is biased. But do not spend too much time trying to prove the editor or referee wrong; simply explain why your manuscript deserves a second look. You should always offer to add new data and not just make superficial changes. Stress that you are willing to do everything and more to alleviate the editors' and referees' concerns and to improve the paper. Point out (politely) what factual errors were made in the interpretations of the data.

What doesn't help (and is more often supplied in rebuttal letters)? Inflammatory language. Calling the editors or referees idiots. Bribes (rare) or threats of varying seriousness (not as rare). Blanket statements that the referees are unfair. Celebrity endorsements like "Nobel laureate X said my paper was great." Cosmetic rewriting of the paper. Guesses at referee identity followed by personal attacks. Statements about your reputation and where you have previously published. And worst of all, don't tell us that we "published a worse paper on a similar subject."

\section{Personal pet peeves}

One of my biggest pet peeves is overuse of the phrase "As you know ..." After nearly seven years as an editor, I feel fairly well versed in scientific facts, but I find every day that there is something more to learn and even more that I do not know. Given that the JCI is a journal that often publishes papers on the minutiae of mechanisms of sometimes obscure diseases, the phrase "as you know" often does not apply. You should never use this phraseology unless the fact is patently obvious (in which case, it probably need not be included). Compare these examples: "As you know, patients with cystic fibrosis can sometimes find it difficult to breathe" and "As you know, excess dietary cholesterol can be used to ameliorate the retinal dysfunction associated with Smith-Lemli-Opitz syndrome (SLOS)." One fact is obvious, one - not so much.

Another irksome phraseology relates to the way diseases are introduced. Clearly the disease under investigation is the most important, but it can be introduced in a way that does not require fancy statistics and superlatives. Set the stage for what is to come and introduce the disease in question without a paragraph of statistics. I found this introduction to a recent study particularly apt, "Osteoporosis is a degenerative bone disease marked by overresorption of bone by osteoclasts." More informative, but entirely excessive, is the following example: "Osteoporosis is a major public health threat for an estimated 44 million Americans, or 55\% of the people 50 years of age and older. In the US, 10 million individuals are estimated to already have the disease and almost 34 million more are estimated to have low bone mass, placing them at risk for osteoporosis." Which would you prefer to preface an article about osteopontin signaling?

Alzheimer disease. Parkinson disease. Lou Gehrig's disease. It is subtle, but can you see the difference an apostrophe can make? Diseases that are named for the scientist who discovered them do not have an apostrophe, while diseases named for a notorious sufferer do use the apostrophe. This has been a long-standing rule, and we have approached PubMed about ensuring that searches with and without the apostrophe will show results for both. Speaking of apostrophes: its versus it's - use it's with an apostrophe when you could substitute "it is." Use its when you refer to possession. It's a simple rule. On a related note, it pays to proofread carefully, as a poorly written study is less likely to impress either the referees or the editorial board. We appreciate the efforts that many of our non-native English speakers make when they engage the services of a professional editor.

Do not start sentences with numbers. Unless you are coining a new "term" do not use quotes. Also never use exclamation points! Not even in the cover letter! Your science is not hot or sexy; do not refer to it as such. The following sentence taken from a recent cover letter gave me palpitations, "As you know, this area of autoimmunity research is quite 'hot' right now!"

Hopefully, this article has given you some useful pointers and reinforced rules that many of you already knew. My hope is that all writers can find some room for creativity with this overall formula for how to submit a successful article and that I didn't put any reader - young or old - to sleep.

\footnotetext{
1. The Scientist Staff. 2007. How to resolve authorship disputes. Scientist. http://www.the-scientist. com/news/display/53485/.

2. Wells, W.A. 2004. Me write pretty one day: how
}

to write a good scientific paper. J. Cell Biol. 165:757-758.

3. Neill, U.S., Thompson, C.B., Feldmann, M., and Kelley, W.N. 2007. A new JCI conflict of interest policy. J. Clin. Invest. 117:506-508.

4. Kahn, C.R., and Roth, J. 2004. Berson, Yalow, and the JCI: the agony and the ecstasy. J. Clin. Invest. 114:1051-1054. 